\title{
Introduction \& Overview to Symposium 240: Binary Stars as Critical Tools and Tests in Contemporary Astrophysics
}

\author{
Edward F. Guinan ${ }^{1}$, Petr Harmanec ${ }^{2}$ and William Hartkopf ${ }^{3}$ \\ ${ }^{1}$ Department of Astronomy \& Astrophysics, Villanova University, Villanova, PA, 19085, USA \\ e-mail: edward.guinan@villanova.edu \\ ${ }^{2}$ Astronomical Institute of the Academy of Sciences of the Czech Republic, 25165 Ondrejov, \\ Czech Republic \\ ${ }^{3}$ Astrometry Dept., U.S. Naval Observatory, Washington, DC, 20392, USA
}

\begin{abstract}
An overview is presented of the many new and exciting developments in binary and multiple star studies that were discussed at IAU Symposium 240. Impacts on binary and multiple star studies from new technologies, techniques, instruments, missions and theory are highlighted. It is crucial to study binary and multiple stars because the vast majority of stars $(>60 \%)$ in our Galaxy and in other galaxies consist, not of single stars, but of double and multiple star systems. To understand galaxies we need to understand stars, but since most are members of binary and multiple star systems, we need to study and understand binary stars. The major advances in technology, instrumentation, computers, and theory have revolutionized what we know (and also don't know) about binary and multiple star systems. Data now available from interferometry (with milliarcsecond [mas] and sub-mas precisions), high-precision radial velocities $(\sim 1-2 \mathrm{~m} / \mathrm{s})$ and high precision photometry $(<1-2$ milli-mag) as well as the wealth of new data that are pouring in from panoramic optical and infrared surveys (e.g., $>10,000$ new binaries found since 1995), have led to a renaissance in binary star and multiple star studies. For example, advances have lead to the discovery of new classes of binary systems with planet and brown dwarf components (over 200 systems). Also, extremely valuable data about binary stars are available across the entire electromagnetic spectrum - from gamma-ray to IR space missions and from the ground using increasingly more powerful and plentiful optical and radio telescopes as well as robotic telescopes. In the immediate future, spectral coverage could even be extended beyond the radio to the first detection of gravity waves from interacting close binaries. Also, both the quality and quantity of data now available on binary and multiple stars are making it possible to gain unprecedented new insights into the structure, and formation and evolution of binary stars, as well as providing valuable astrophysical information (like precise stellar masses, radii, ages, luminosities and distances) to test and constrain current astrophysical theory. These major advances permit tests of current theories and ideas in stellar astrophysics and provide the foundations for the next steps in modeling and improvements in theory to be taken.
\end{abstract}

\section{Introduction}

IAU Symposium 240, Binary Stars as Critical Tools 8 Tests in Contemporary Astrophysics, took place 22-25 August 2006, during the second week of the XXVI ${ }^{\text {th }}$ IAU General Assembly in Prague, Czech Republic. The meeting lasted for 3-1/2 days and consisted of a mix of invited, contributed oral contributions and over 180 posters. This symposium was the result of a merger of two binary star symposia proposals developed for the General Assembly. One proposal, originated from Commission 26 (Binary and Multiple Stars) and led by Hartkopf, focused mainly on wider systems. The other proposal was organized by Commission 42 (Close Binaries) and led by Harmanec and Guinan, and it focused primarily on close/interacting binaries. The IAU Executive Committee 
recommended that the two proposals be merged, and after hundreds of e-mail exchanges between the wide and close binary groups and their respective organizing committees and related commissions, the present symposium was organized with a broader program that includes all varieties and flavors of binary stars - both close and wide as well as near and far binaries and multiple stars systems. Most of the different types of binary systems discussed at the symposium are given in Figures $1-3$. The various classes of binary systems composed of non-degenerate stars are shown in Figure 1, while Figure 2 shows binaries containing at least one degenerate component (white dwarf, neutron star or black hole). Figure 3 shows recently discovered binary systems with planetary or brown dwarf components. Note that our solar system would be included in Figure 3.

The symposium brought together $\sim 500$ astronomers from 54 countries who are involved in all aspects of binary and multiple star research, from very long-period, common-propermotion pairs and other "fragile" binaries to short-period contact binaries, short-period binaries with degenerate components, as well as star/brown-dwarf/planet systems, with the aim of exploring interests common to all binary star researchers. Both the observational and theoretical aspects of binary and multiple star research are represented, but the main themes of the program are the new information and physical insights gleamed from the recent advances in instrumentation and techniques. The meeting also attracted those interested in the observational and theoretical aspects of modern stellar astrophysics that depend very strongly on the fundamental properties of stars found primarily from binary and multiple stars. Sponsored by Division IV [Stars] and Commissions 26 and 42, the symposium has also received strong support from Division V [Binary \& Variable Stars], as well as from seven other commissions and three working groups. We thank these Divisions, Commissions, and Working Groups for their support.

The resulting program benefited greatly from the infusion of new ideas and differing perspectives from the two binary star communities, which resulted in a broader and more comprehensive program and most of all a more vibrant and interesting meeting. The format for the symposium was a mix of invited oral review presentations $(\sim 30 \mathrm{~min})$ and more narrowly-focused topical $(\sim 15-20 \mathrm{~min})$ presentations. There were also over twenty short oral/poster presentations $(5-10 \mathrm{~min})$ that were selected by the SOC from $>180$ submitted posters. These are also included in this volume, as are abstracts of all the poster presentations posted to the publisher's website.

\subsection{Venue and dedication of the symposium}

It is fitting that this symposium was held in the Czech Republic, because much of the pioneering work on binary and variable stars has been carried out in Central and Eastern Europe for over a century. The meeting was also fittingly held in the city of Prague, home to both Tycho Brahe and Johannes Kepler. It is noteworthy that Tycho carried out the first accurate astrometric measurements, while Kepler's laws of orbital motion play such an important role in binary star studies. As the first joint meeting of the "close and wide" binary communities in recent memory, it was also appropriate to jointly dedicate the symposium to outstanding representatives of both of those "close" and "wide" worlds of binary and multiple star research: Mirek Plavec of the Czech Republic and the late Charles Worley of the United States. Both gentlemen attended the last General Assembly held in Prague in 1967, and Plavec also attended the opening session of the 2006 meeting. Poster papers about both astronomers, displayed at the meeting and included here in these proceedings, provide brief descriptions of their professional careers as well as their many important contributions to binary and multiple star research.

Also, the reader should take note of the poster paper by Augensen, Mason \& Hartkopf (see publisher's website) discussing the life and work of Dr. Wulff Heintz, who passed 


\section{Binary Systems with Non-Degenerate Components}

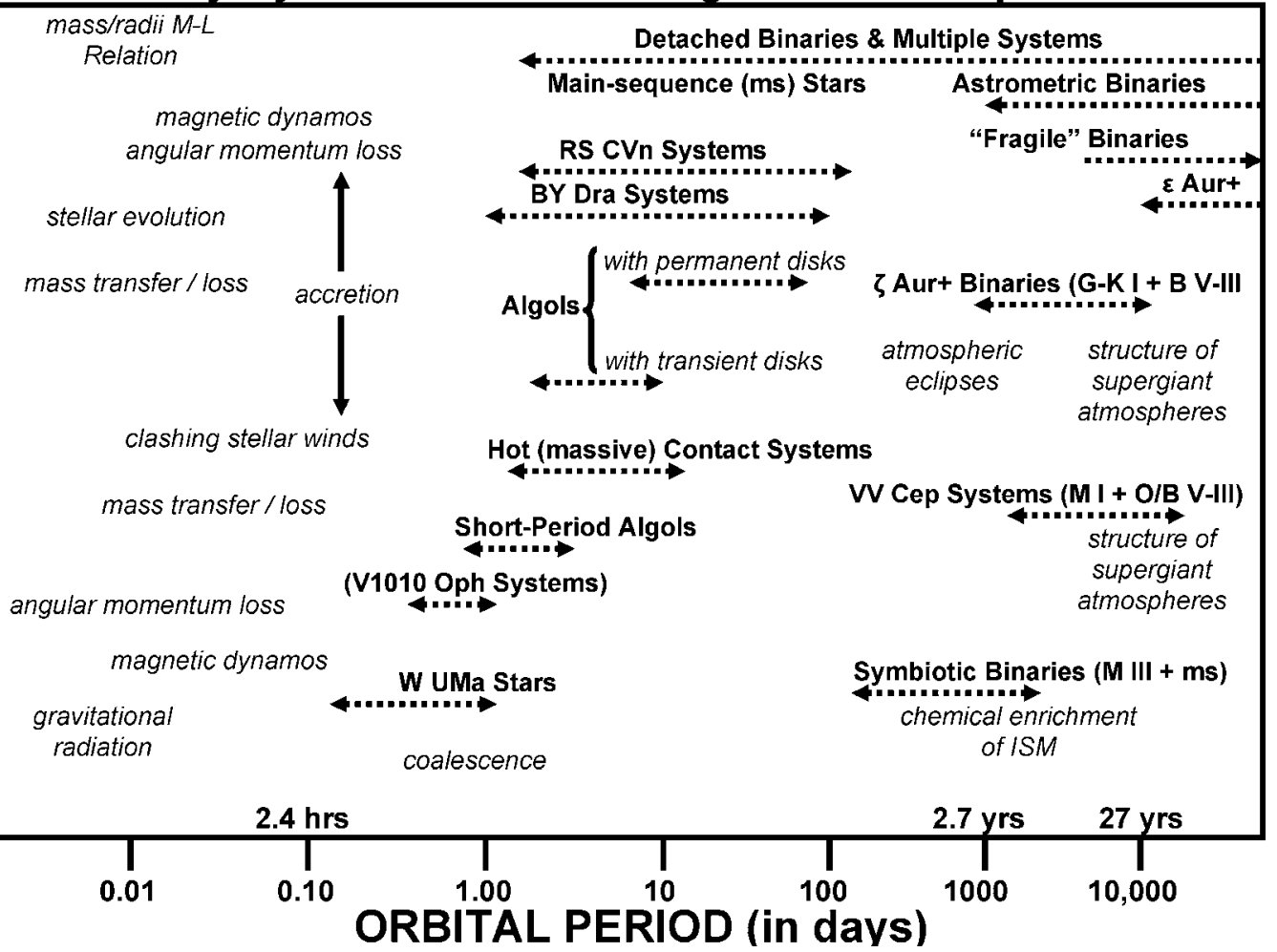

Figure 1. The period range and astrophysical interests of binary systems with non-degenerate components.

away in June 2006, shortly before this symposium was held. Noteworthy among Wulff's many contributions to binary and multiple star research is that he made a total of 54,000 micrometer measures of double stars and discovered over 900 new pairs. Wulff is second only to Willem van den Bos in the total number of astrometric measures. Interesting enough, Charles Worley is in third place in this category, with over 40,000 individual astrometric measures and the discovery of 39 new faint, cool stellar companions.

\section{Background: Major Advances in Instrumentations and Techniques}

In 1992, IAU Colloquium 135, "Complementary Approaches to Double and Multiple Star Research," was held at Callaway Gardens (near Atlanta, Georgia), and was meant to emphasize the expanding overlap of observational opportunities offered to binary star researchers by advances in precise radial velocity techniques, interferometry, etc. Additional topics included recent advances (for those times) in our knowledge of duplicity for young stars and pre-main sequence stars, the latest theories of binary and multiple star formation, and the tantalizing first results from HST and Hipparcos. The meeting successfully brought together nearly one hundred astronomers with a diversity of expertise until then rarely found at a single meeting on binary stars.

Much has changed in the ensuing 14 years! HST is now approaching the end of its life, and its larger successor, the James Webb Space Telescope (JWST) is under construction. Hipparcos results have been published and well-studied, and the next generation of astrometry satellites (such as the Space interferometry Mission [SIM] and Gaia) now 
Binary Systems with Degenerate Components

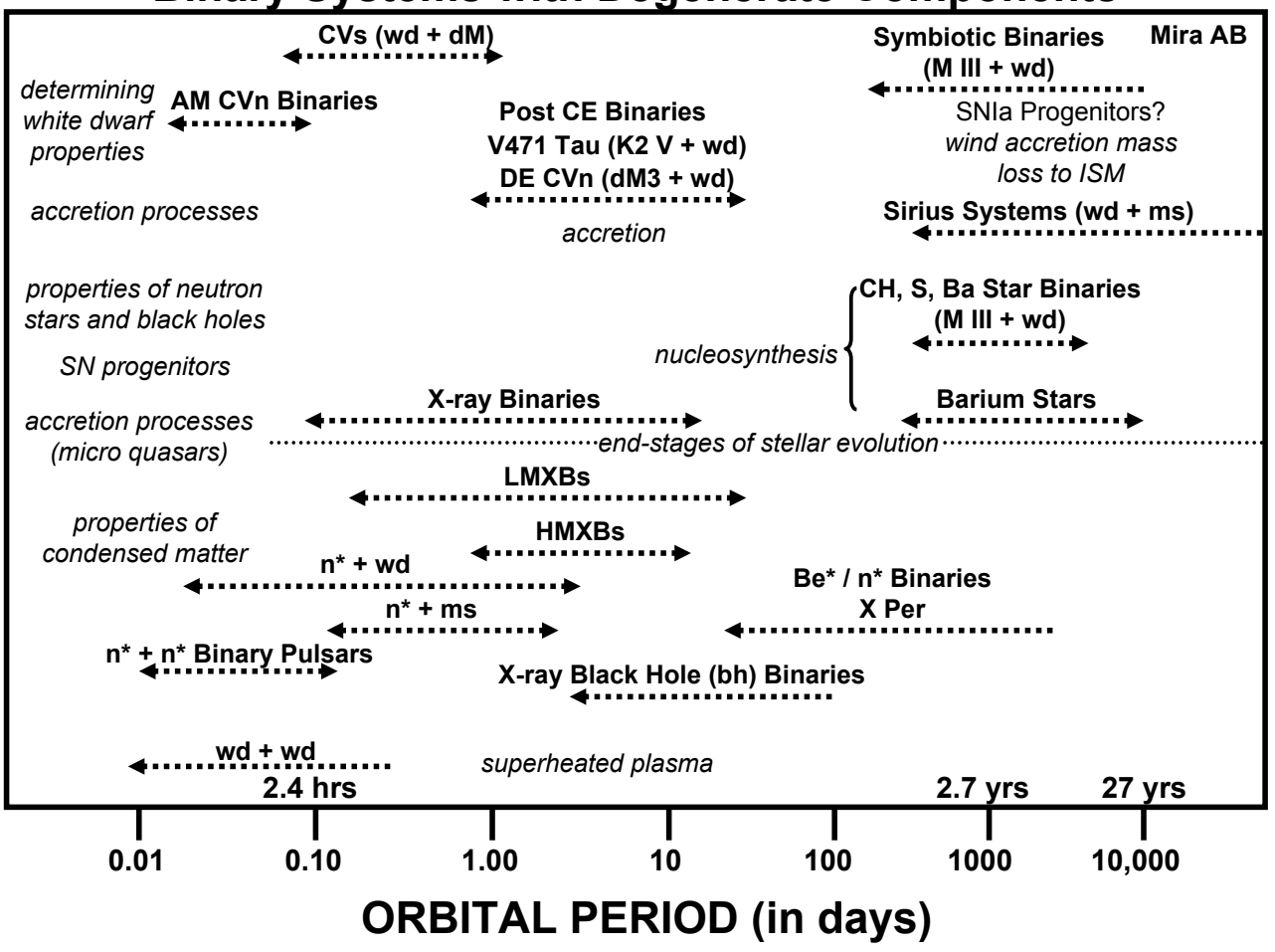

Figure 2. The period range and astrophysical interests of binary systems with degenerate components.

under development promise results orders of magnitude greater in both precision and quantity. Speckle interferometry and adaptive optics have replaced visual micrometry as the routine methods of measuring visual binaries, while long-baseline interferometry has now produced a significant body of results for closer binaries, with the promise of an outburst of activity in the resolution of spectroscopic binary systems during the next few years. From astrometric and spectroscopic orbits, masses and other important stellar properties are determinable. Interferometric results from the HST Fine Guidance Sensors (FGS) have also become more plentiful. As discussed in several papers at the meeting and in this volume, there have been dramatic changes in the way we conduct astrometry of binaries and profound increases in the reliability of the results.

Similar tremendous advances continue in other areas as well. Variability-induced motion and other color-based detection methods are being used to mine the SDSS database for new binaries and may be used with Gaia (http://www.rssd.esa.int/index.php? project $=$ GAIA\&page=index) and SIM (http://planetquest.jpl.nasa.gov/SIM/ sim_index.cfm) data as well. Superior infrared detectors and techniques, as well as the recent availability of infrared spectroscopy and imaging from space with the Spitzer Space Telescope (SST), are revolutionizing the study of pre-main sequence binaries and binaries with cool star and brown dwarf components. Ever more accurate radial velocity techniques have yielded long-period orbits that further blur the distinction between the traditional spectroscopic and visual separation/period regimes and yield accurate stellar masses across the spectral range - not to mention the new short-period systems and $200+$ exoplanets discovered over the last decade! Within the next several years hundreds 


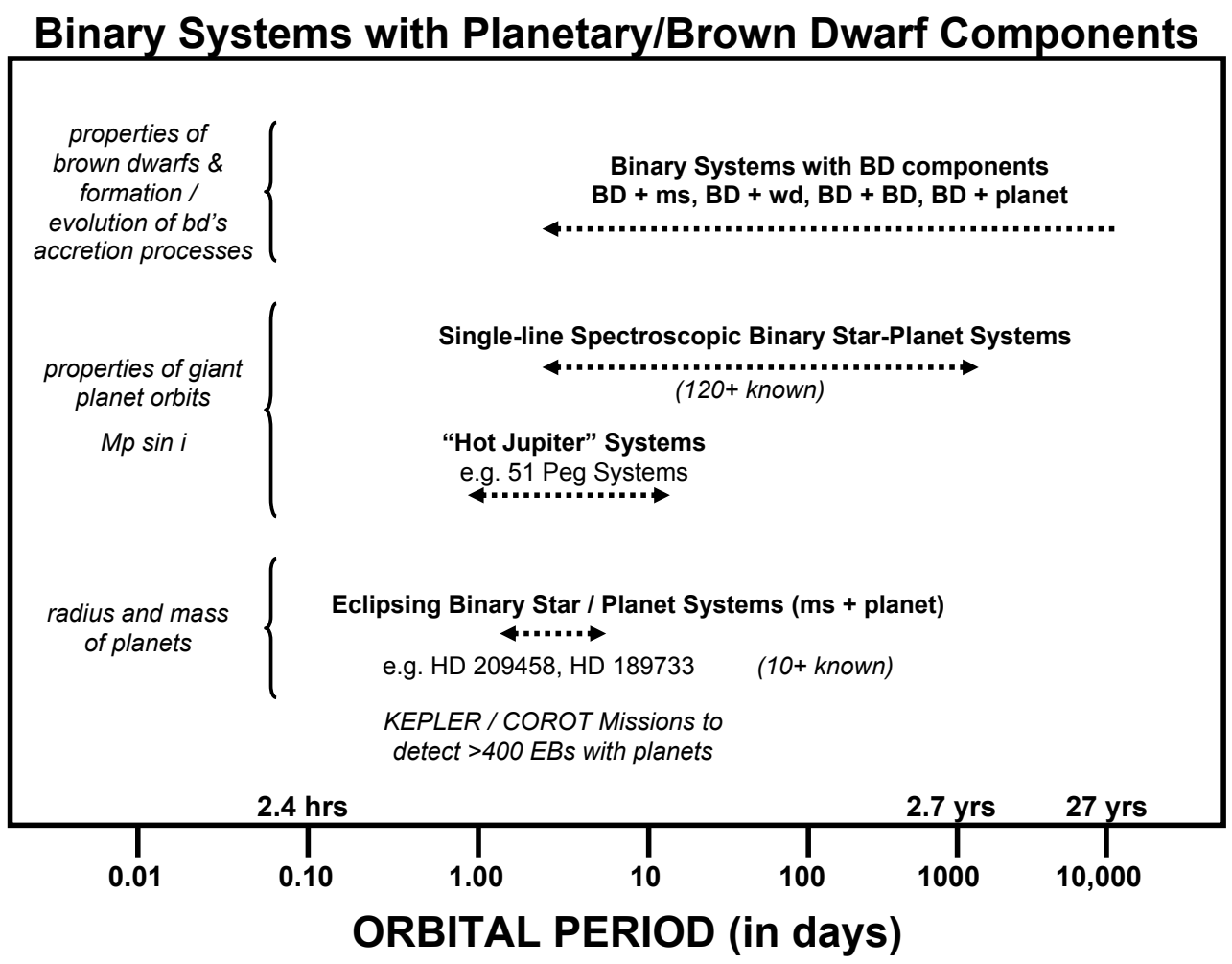

Figure 3. The period range and astrophysical interests of binary systems with brown dwarf and planetary components.

of additional exoplanets (some with diameters as small as the Earth) are expected to be discovered by the CoRot and Kepler missions.

\subsection{Organization of the papers}

The papers in this volume are organized into three major themes, as discussed in Sections 3-5 below. "New Observing Techniques and Reduction Methods" includes (3.1) Observing with high angular and spectral resolution; (3.2) New possibilities for standard observational techniques; (3.3) Improved methods of data analysis; and Observing in the era of large scale surveys. "Binary Stars as Critical Tools (for obtaining direct and reliable Astrophysical Information)" consists of (4.1) The need to improve basic calibrations and increasing possibilities of classical methods and (4.2) Evolutionary models for binary and multiple stars. Finally, "Binary Stars as Critical Tests" focuses on (5.1) Binary stars as probes of our Galaxy and (5.2) Asteroseismology and Stellar Activity. The volume concludes with excellent summaries on the observational aspects (given by Colin Scarfe) and on the theoretical aspects (given by Virginia Trimble) and a few closing comments from Hartkopf. The papers (both oral presentations and posters) presented at this symposium and included in this volume and on the publisher's website demonstrate that these are indeed very exciting times for the studies of binary and multiple star systems. 


\section{New Observing Techniques and Reduction Methods}

\subsection{Observing with high angular and spectral resolution}

Recent results from powerful long-baseline interferometers both in the northern and southern hemispheres are discussed by Hal McAlister and John Davis. These instruments are yielding unprecedented high angular resolution measures, and refined astrometric orbits, of an increasing number of binaries with smaller and smaller separations. These papers show that astrometric measures are now closing the gap between wide binaries and shorter-period spectroscopic binaries and are providing fundamental properties of the component stars not possible previously except for eclipsing systems. In many cases additional components have been discovered to single stars and doubles. As discussed by Yuri Balega, interferometry using single telescopes (mostly from speckle interferometry), is discovering many new binaries and multiple star systems, and these observations are routinely yielding high precision orbits. Also with interferometric and adaptive optics techniques, many single and binary stars are being found to harbor fainter stellar and, in some cases, brown-dwarf and planetary-size companions. As discussed by Mike Shao and Xiaopei Pan, the Space Interferometry Mission (SIM), currently planned for 2015, will be capable of ultra-high angular precision, microarcsecond ( $\mu$-sec) astrometry that has the capability of astrometrically detecting components with masses as small as Earth. In a contributed oral/poster presentation, Frank Fekel \& Jocelyn Tomkin discuss several spectroscopic binary candidates that are most suitable for ground-base multiple aperture interferometry.

A decade ago, most astronomers (including most members of Commission 42) would have been very happy with stellar radial velocity measures with precisions of $\approx 1-3 \mathrm{~km} / \mathrm{s}$. However, over the last decade radial velocity measures using, for example, iodine gas cells, are routinely being obtained with precisions of better than $5 \mathrm{~m} / \mathrm{s}$. More recently, radial velocity measures are being accomplished with precisions reaching as low as $\sim 1 \mathrm{~m} / \mathrm{s}$. As discussed at the meeting by Artie Hatzes (but not included in the book) and others, over 200 extrasolar planets have so far been discovered, mostly from these highprecision radial-velocity programs (see, e.g., http://exoplanets.org). It appears that $\sim 5 \%$ of nearby solar-type (i.e., $\mathrm{dG}$ and $\mathrm{dK}$ ) stars are accompanied by Jupiter-size planets, many of these (known as "Hot Jupiters") are orbiting very close to their host stars. We have not yet reached the needed observational precision to detect Earth-size planets from high-precision spectroscopy but, as reported by several papers here, Earthsize planets should soon be discovered with SIM astrometry. These discoveries may come more immediately from high-precision photometry missions such as CoRot and Kepler, however, as discussed later in the book. Excellent summaries of high-precision radial velocity work are given by Marcy \& Butler (http://exoplanets.org/) and Mayor (http://obswww. unige.ch/ udry/planet/). For the most part, the high-precision radial velocity work is focused on finding planets. However, in the near future, these measures will also be secured for double-line eclipsing binaries to obtain very accurate fundamental properties.

\subsection{New possibilities for standard observational techniques}

It is commonly thought by many stellar astronomers that observing time on large telescopes is impossible to get because this precious time is monopolized by extragalactic astronomers. As discussed in papers by Ignasi Ribas and Alceste Bonanos, however, this is clearly not the case. It is still possible to get observing proposals on binary stars passed by the scrutinizing eyes of TACs guarding the time on large telescopes. Ribas focuses mainly on spectroscopic observations of faint, but astrophysically important, eclipsing 
binaries that are members of Local Group galaxies (mostly O/B binaries) and intrinsically faint low-mass red-dwarf and brown-dwarf eclipsing systems. Bonanos discusses observations of O/B eclipsing binaries in the Local Group and how these stars are providing independent, accurate distances to the Large and Small Magellanic Clouds, M31 and her recent work on determining an accurate distance to the Triangulum spiral (M33). There are a number of posters on these topics presented at the meeting.

Brian Mason discusses the results of (in some cases) over a hundred years of standard (or classical) astrometric observations of visual and multiple stars and presents some interesting results and statistics. As demonstrated by previous papers, many additional fainter members of binary and multiple star systems are being discovered in increasing numbers, thanks to the advances in instrumentation and technologies (mostly from interferometry, adaptive optics and high precision spectroscopy). Hartkopf reports on efforts to meet this problem of success in his paper about the nomenclature of multiple stars in the Washington Multiplicity Catalog (WMC; http://ad.usno.navy.mil/wds/wmc.html).

Also included here are several interesting oral/poster contributions. These papers focus on a wide range of topics that include a new study and mass determination of the Polaris multiple star system (Nancy Evans et al.), a new and very interesting eclipsing binary (DE CVn) consisting of a dM3 star and a cool white dwarf that must have evolved through the common-envelope stage of binary star evolution (Else van den Besselaar et al.), a new study of the eclipsing triple system U Oph (Luiz Paulo Vaz et al.) and a new study of the very young binary stars in the Orion Nebula Cluster (Rainer Köehler et al.). Additionally, there is a related poster paper on DE CVn by Ümit Goker \& Günay Tas. Also here, Petr Hadrava discusses new trends in disentangling the spectra of multiple stars using methods that he pioneered.

\subsection{Improved methods of data analysis}

Powerful and improved methods for the analysis of binary and multiple stars, such as spectra disentangling, Doppler tomography, Doppler imaging and eclipse mapping, as well as other innovative modeling procedures are the primary themes of this section. Herman Hensberge \& Krešimir Pavlovski present detailed discussions of the modern analysis techniques now being applied mainly to spectroscopic binaries. They discuss the advantages and disadvantages of the different methods to extract precise radial velocity information from spectroscopic binaries. Most of the emphasis is on spectra disentangling and the information gained. Mercedes Richards discusses Doppler Tomography as applied to mapping streams and disks in Algol systems, while Klaus Strassmeier discusses Doppler Imaging and constructing eclipse maps of spots and other stellar surface features on chromospherically active components of close binary systems — such as RS CVn stars.

In a thoughtful paper by the developer of the most widely used program for analyzing and modeling eclipsing binary light curve data, Bob Wilson focuses mainly on advances and innovations in light curve modeling developed over the last three years. He also looks to the future and discusses methods for the modeling of the tens of thousands of light curves of eclipsing binaries expected over the next several years. Wilson advocates publication of future lightcurves of eclipsing binaries not in delta-mags, but rather in physical units such as ergs $/ \mathrm{sec} / \mathrm{cm}^{2}$. This is easier said than done, but would allow direct distance estimations within the light and radial velocity curve modeling.

In the same section, Theo ten Brummelaar discusses the practicalities of reducing multi-aperture interferometric data. Applications of improved methods of binary star analysis are presented by Geraldine Peters in her study of the bipolar jets, hot interacting regions and colliding winds in OB interacting binaries, and by Styliani Kafka on the 
evidence for chromospheric activity on the cool, low mass secondaries of cataclysmic variable binaries.

In an oral-only presentation, Dimitri Pourbaix discussed the use of large-scale surveys for discovering and studying new binary stars. A good example of this is found in the multi-band astrometry detection of binaries from the Sloan Digital Sky Survey (SDSS). These binaries are detected from the astrometric color-induced displacement technique (CID) in which the displacement of the photocenter between different bandpasses arises from a varying contribution of differently colored components to the total light. (see Pourbaix et al. 2004, A\&A, 423, 755 for more details.)

Several interesting contributed papers appearing are the contributions of Diego FalcetaGonćalves et al. on the massive binary/multiple star system $\eta$ Carinae and the paper by Bob Stencel about another favorite binary - the 27-yr eclipsing (F2I + disk) binary, $\epsilon$ Aurigae. It is noteworthy that $\epsilon$ Aur is scheduled to start its next $\sim 2$ year-long eclipse in 2009. Another famous (infamous?) binary to join $\eta$ Car and $\epsilon$ Aur in this section is $\beta$ Lyr. Pavel Chadima and company analyzed hundreds of spectra for this perplexing binary and, using the KOREL program, found strong evidence that supports mass accretion and the presence of a thick accretion disk with bi-polar jets, as well as a circumbinary envelope. It will be interesting in the future to image $\beta$ Lyr with the next generation of multiaperture interferometers. This session has a great number of interesting posters worth visiting.

As discussed in the papers by Wilson, Ribas and others in this section, it will not be long before high-precision radial velocity data will be combined with high precision $(\sim 1$ mmag) photometric light curves of selected double-line eclipsing binaries, yielding the masses and radii of their component stars with uncertainties better than $\sim 0.2 \%$. Now most stellar masses and radii are known typically to no better than $\sim 5 \%$. Examples of the high precision light curves of eclipsing binaries that are now even possible using the star tracker of the WIRE satellite are given by Hans Bruntt et al. The Canadian MOST microsatellite (http://www.astro.ubc.ca/MOST/) also currently has the capability of millimag photometry, but only a few eclipsing binaries have been observed so far. As discussed in the next section, light curves, with even higher precisions (better than $0.1 \mathrm{mmag}$ ) are expected very soon with the CoRot and Kepler space missions.

\subsection{Observing in the era of large-scale surveys}

Another important development discussed in a number of papers and posters at this meeting is the bonanza of new (mainly eclipsing) binaries discovered over the last decade from photometric survey and monitoring programs. Most of these binaries have been serendipitously discovered from micro-lensing photometry programs such as EROS, OGLE, and MACHO. So far several thousand new eclipsing binaries have been found, mostly in the rich star fields of the Galactic Bulge and the Magellanic Clouds. Also, nearly a thousand additional eclipsing binaries have been discovered by the DIRECT program (see Bonanos' paper) and by Ribas and collaborators in the M31 and M33 spiral galaxies. Some of these newly discovered extragalactic eclipsing binaries have been used to secure accurate distances to the Local Group galaxies (see also several posters in this section). A brief inventory of the eclipsing binaries found so far in Local Group galaxies is given in Figure 4.

But in the near future, thanks to planned ground-based panoramic photometric surveys such as Pan-STARRS (http://www.pan-starrs.ifa.hawaii.edu/) and the Large Synoptic Survey Telescope (LSST; http://www.lsst.org./) and several other programs, the numbers of eclipsing binaries are expected to swell to over $10^{5}$ systems. How do we cope with such an abundance of riches? Several groups are trying to find the solution to 


\section{ECLIPSING BINARIES IN THE LOCAL GROUP GALAXIES}

\begin{tabular}{|c|c|c|}
\hline Galaxy & \# of known EBs & Notes \\
\hline The Milky Way & 4000 (Many in Bulge) & Traditional photometry \& OGLE \\
\hline LMC & $5000^{+}$ & EROS, OGLE, \\
\hline SMC & $2000+$ & MACHO \& MOA \\
\hline $\begin{array}{l}\text { M31 } \\
\text { M33 }\end{array}$ & $400+$ & $\begin{array}{c}\text { DIRECT Project } \\
\text { (see Bonanos - this volume) } \\
\text { (also Ribas - this volume) } \\
>10^{5} \text { expected at } \\
m \sim 24^{\text {th }} \text { mag } \\
\text { (Bonanos - this volume) }\end{array}$ \\
\hline Fornax & $\sim 15$ & $\begin{array}{l}\text { Measured from } 1 / 8 \mathrm{CCD} \text { chips of } \\
\text { mosaic; (extrapolated value from mosaic: } \\
\sim 300 \text { ) (Clementini - priv. comm.) }\end{array}$ \\
\hline Leo I & $\sim 10+$ & $\begin{array}{c}\text { Down to } 24^{\text {th }} \text { mag. Only short period (P } \\
<2 \text { days) discovered so far. }\end{array}$ \\
\hline NGC 6822 & $6^{+}$ & $\begin{array}{c}\text { Only } 25 \% \text { of galaxy covered (Clementini } \\
\text { \& collaborators) }\end{array}$ \\
\hline Carina $(\mathrm{dSp})$ & $5+$ & Carina Project (Monelli \& collaborators) \\
\hline
\end{tabular}

Figure 4. Eclipsing binaries in local group galaxies.

this by developing automated methods to analyzed thousands of light curves at a time. Andrej Prša \& Tomaž Zwitter discuss a very promising pipeline reduction method of analyzing large numbers of light curves, while Tsevi Mazeh and colleagues discuss their automatic approach and initial results when applied to the study of early-type binaries in the Magellanic Clouds. Their preliminary results indicate that, at least among B-type stars, binaries may be far less frequent in the LMC $(<10 \%)$ as compared to similar stars in the solar neighborhood. There are posters and related papers at the conference by Jonathan Devor \& David Charbonneau and by Aliz Derekas et al.

In the same session there are fascinating papers by David Koch (and the Kepler team) about the ultra-high precision photometry expected from the Kepler mission and a related paper by Carla Maceroni and Ribas on the impact of very high precision photometry on close binary stars expected shortly from the CoRot space experiment (http://www.esa.int/science/corot/). These missions are expected to discover hundreds of additional planetary transit systems. Currently, about a dozen eclipsing planetstar systems have been uncovered. Also there are short papers presented by Panos Niarchos et al. on the Gaia mission's impact on binary star studies. Other interesting papers include the results of multiplicity studies of very young Herbig Ae/Be stars (Sandrine Thomas et al.) and the results of a multiplicity study in nearby solar-type stars (Deepak Raghavan et al.), as well as a contributed paper on the feasibility of detecting planets in wide binaries from ground-based Adaptive Optics (AO) systems (Ralph Neuhäuser et al.). 


\section{Binary Stars as Critical Tools}

In this section many examples are provided of using binary and multiple stars as critical tools for studying a wide variety of important astrophysical problems. There are a number of interesting examples discussed in the papers included here.

\subsection{The need to improve basic calibrations using binary stars and increasing the possibilities of classical methods}

Jason Aufdenberg and colleagues report on the recent images of the rotationally distorted and gravity darkened bright stars made with the longest baselines of the CHARA Array and the Fiber-Linked Unit for Optical Recombination (FLUOR). They discuss their previous result on imaging the pole-on rapid rotator Vega, in which the star is clearly resolved and imaged. Here they also discuss the most recent results on imaging the components of the 4-day, non-eclipsing, double-line binary Spica. For Spica, in addition to resolving and imaging the individual components of the close binary, they discuss improvements in determining its apsidal motion by combining their observations with previous interferometry measures. In the same session Alvaro Giménez discusses the apsidal motion studies of eccentric eclipsing systems as a powerful means of studying the interiors of the stellar components. Except for a few outstanding cases, the internal structure determined from apsidal motion studies agrees very well with stellar evolution and stellar structure theory. In another important talk, David Valls-Gabaud discusses the results of using different methods to secure a reliable distance to the Pleiades Cluster. In summary, binary stars are yielding the most reliable Pleiades distance of about $134 \pm 3 \mathrm{pc}$. (It should be recalled that several years ago Hipparcos astrometry returned a distance of only $118 \pm 3 \mathrm{pc}$ for the Pleiades.) Thanks to binary stars, the distance to such an important cluster is now reliably known.

Todd Henry focuses on a decade-long endeavor to calibrate the Mass-Luminosity Relation (MLR) for the numerous low-mass and low-luminosity red dwarfs and brown dwarfs that make up over $70 \%$ of the stars in our Galaxy. To get an accurate measure of these faint but numerous stars and substars, they have to be members of binary systems. He focuses on efforts to calibrate the MLR for dM stars as well as T and L dwarfs. In this talk (the abstract of which is included in this volume) he gives these dim, low-mass stars the respect they deserve, since these stars may be the dominant contributor to baryonic mass in the Universe. Ben Lane discussed recent developments in the study of extrasolar planet systems, including work in nulling interferometry and the Palomar High-precision Astrometric Search for Exoplanet Systems (PHASES); unfortunately his talk is not included in these proceedings.

In another interesting and thought-provoking paper, Terry Oswalt discusses the use of the loosely bound "fragile" binary stars to investigate several astrophysical problems. One interesting aspect featured in this paper is using the old fragile binaries to probe the dynamics and early history of our Galaxy and also to use white dwarf + main sequence star binaries to better calibrate the cooling times of white dwarfs or use the cooling ages of white dwarfs to study the properties of its companion.

Other interesting contributed papers in this session are papers dealing with the diverse topics that include the direct measures of tidal dissipation in highly eccentric binaries (Andrei Tokovinin), a study of the famous cataclysmic binary U Gem (Juan EchevarríaRoman), the study of "runaway" stars (M. Virginia McSwain et al.) and an interesting paper on "mining" catalogs of common proper motion binaries (Julio Chanamé). Also not to be missed are over a dozen related poster papers. 


\subsection{Evolutionary models for binary and multiple stars}

Cathy Clarke opens this session with a thorough review of the major advances in the theories of binary star formation that have been made over the last several years. She concludes that results of current binary star formation theories and simulations, in which star formation occur in high interactive modes, appear to fit the observed binary and multiple star characteristics and frequencies fairly well. However, there are still problems with an agreement between theory and observations when it comes to low mass stars and in the tendency for models not to produce enough binaries with low mass ratios. In a related paper Peter Eggleton and colleagues discuss the incidence of multiple stars among bright stars found in bright star catalogs. They have identified over 4500 such systems and subdivided them in terms of frequencies of multiplicity and distributions of periods and sub-periods. In this study they discuss the results of their Monte-Carlo simulations to generate systems with roughly the observed multiplicities and orbital properties of the stars in this sample.

In another interesting paper Hans Zinnecker presents the study of young binary stars as tests of pre-main sequence evolution tracks. Some fascinating examples of various type of binaries with PMS components are included in the paper. He finds that with a sufficiently large sample of different masses and ages of resolved PMS SB2 systems, that most of the parameter space of pre-main sequence evolution tracks can be tested. In an interesting theoretical study Dmitry Bisikalo \& Takuya Matsuda discuss recent progress in the theory and modeling of mass exchange in close binaries. In this paper they present and discuss the 3-D gas dynamic models used in the gas flow and exchange simulations and reach some important conclusions. Also included in this session are contributed poster/oral papers on the study of eclipsing binaries in the Large Magellanic Cloud and the astrophysical quantities that can be determined from them (Derekas et al.) and a study of the initial-final, mass relationship of white dwarfs in common proper motion pairs and in open clusters (Silvia Catalán et al.). Also included is a short contribution about the detectability of planets in wide binaries using ground-based relative astrometry with Adaptive Optics techniques (Neuhäuser et al.). They conclude that it is feasible to detect the small astrometric wobble due to a planet using their method. They have carried tests of the technique using the VLT and obtained astrometric precisions as small as $50 \mu$ as!

\section{Binary Stars as Critical Tests}

It is well known that binary and multiple stars provide critical tests of the structural and evolutionary theories of stars but, as shown by the papers here, binary stars are also important as tests of galactic evolution and dynamics, as well as providing testbeds for stellar pulsation theory \& asteroseismology, along with studying magnetic activity in close binaries with cool star components.

\subsection{Binary and multiple stars as probes of our Galaxy}

This section contains a number of very interesting and diverse papers and posters covering a wide range of phenomena that include binary stars as probes of our Galaxy, asteroseismology, stellar activity and magnetism. Included are papers on general themes of "Binary Stars of our Galaxy" in which Dany Vanbeveren and Erwin De Donder discuss how mass loss from the final stages of some close binary stars plays a major role in the chemical enrichment and evolution of our Galaxy (and presumably in other galaxies as well). On the related subject of binary stars as probes of the Galaxy are the papers by 
Christine Allen, Arcadio Poveda and Helmut Abt that focus primarily on using the orbital properties of wide binaries to study binary star evolution, as well as the dynamical history and evolution of our Galaxy. When statistically analyzed, the frequency of these wide binaries with differing gravitational bindings and ages yield important clues about the early dynamical history of our Galaxy.

\subsection{Asteroseismology and stellar activity in binary stars}

In a comprehensive review paper by Conny Aerts, an overview of the current status of asteroseismology is provided. She points out the advantages of having a pulsating star as a member of a close binary - in particular as a member of an eclipsing binary. The important new things that can be learned from the study of pulsating components in close binaries are discussed. For example, if the pulsating star is a member of a double-line eclipsing binary, then the physical properties of the pulsating star (radii and masses) are determinable from the traditional analyses of the light and radial velocity curves. Securing reliable physical properties of pulsating stars (such as masses, radii, and luminosities) are critical for the calibration and interpretation of asteroseismic observations expected very shortly from CoRot. Several contributed poster papers also discuss the related issues of stellar pulsation and asteroseismology. For example, Andrzej Pigulski \& Grzegorz Pojmański discuss the discovery of four $\beta$ Cephei variables in eclipsing binary systems. As pointed out by the authors these binaries are very attractive objects for much-needed follow-up spectroscopic and multicolor standard photometry which will yield accurate physical properties of the stars. Once the masses and radii of these stars are reliably known, then their interiors can be probed by means of asteroseismology methods. The reader should check the poster papers on related topics.

Another important topic covered here is the magnetic activity frequently found in close binary systems with cool stars (i.e., stars with outer convective zones). Katalin Oláh's paper discusses the influence of binarity on stellar activity - such as star spots and chromospheric emissions - and finds evidence that some stars in close binaries show stronger manifestations of magnetic activity when compared to single stars with similar physical properties and rotations. On the related subject of stellar magnetic activity, Heidi Korhonen \& Silvia Järvinen discuss magnetically-active stellar longitudes and the rapid changes (flip/flops) that are commonly seen. This behavior may be related to the reversal of the Sun's magnetic field over its $\sim 22$ year activity cycle. There are a number of interesting poster papers on the topic of stellar activity and stellar magnetism in close binaries that should not be overlooked in the poster papers.

\section{Acknowledgements}

This work was sponsored in part by grants from the U.S. National Science Foundation and NASA, which we gratefully acknowledge. We are also grateful to the U.S. Office of Naval Research Global (grant N00014-06-1-1054) for the support of travel costs for some 25 symposium participants, including WIH. EFG would like to acknowledge travel support from a National Science Foundation grant to the American Astronomical Society, and we would like to thank Scott G. Engle of Villanova University for his help in the preparation of this paper. 